\title{
A new technique to efficiently select Compton-thick AGN
}

\author{
P. Severgnini, A. Caccianiga, and R. Della Ceca
}

INAF - Osservatorio Astronomico di Brera, Milano, via Brera 28, 20121 Milano, Italy

e-mail: paola.severgnini@brera.inaf.it

Received 4 November 2011 / Accepted 18 April 2012

\begin{abstract}
Aims. We present a new efficient diagnostic method, based on mid-infrared and X-ray data, to select local $(z<0.1)$ Compton-thick AGN with the aim of estimating their surface and space density.

Methods. We define a region in the X-ray-to-mid-IR $\left[F(2-12 \mathrm{keV}) / F_{25} v_{25}\right]$ vs. X-ray color (HR) plane associated to Compton-thick AGN, i.e. $\left[F(2-12 \mathrm{keV}) / F_{25} v_{25}\right]<0.02$ and $\mathrm{HR}>-0.2$. On the basis of this selection method we build up a sample of 43 Comptonthick AGN candidates using data from IRAS Point Source and 2XMM-Newton catalogues. In order to test the efficiency of the proposed method in selecting Compton-thick AGN we use the results of the X-ray spectral analysis performed on all the sources of our sample (presented in a parallel work). After taking into account the different selection effects, we have estimated the number of Compton-thick in the local Universe and their density down to the IRAS flux limit of $F_{25}=0.5 \mathrm{Jy}$.

Results. We find that the diagnostic plot proposed here is an efficient method to select Compton-thick AGN in the nearby Universe since $\sim 84 \%$ of the sources populating the proposed Compton-thick region are actually Compton-thick AGN. Twenty percent are newly-discovered Compton-thick AGN. We then estimate the surface density of Compton-thick AGN down to the IRAS PSC catalogue flux limit $\left(F_{25}=0.5 \mathrm{Jy}\right)$ that turns out to be $\rho^{\mathrm{CT}} \sim 3 \times 10^{-3} \mathrm{src} \mathrm{deg}^{-2}$. After estimating an equivalent IR-to-hard-X-ray limiting flux, we compare our result with those found with Swift-BAT. We find that the surface density derived here is a factor 4 above the density computed in the hard X-ray surveys. This difference is ascribed, at least in part, to a significant contribution $(\sim 60-90 \%)$ of the star-forming activity to the total $25 \mu \mathrm{m}$ emission for the sources in our sample. By considering only the $25 \mu \mathrm{m}$ AGN emission, we estimate a surface density of Compton-thick AGN which is consistent with the results found by hard X-ray surveys. Finally, we estimated the co-moving space density of Compton-thick AGN with intrinsic $L_{\mathrm{X}}>10^{43} \mathrm{erg} \mathrm{s}^{-1}(0.004<z<0.06)$ : $\Phi_{\mathrm{C}-\text { thick }} \sim\left(3.5_{-0.5}^{+4.5}\right) \times 10^{-6} \mathrm{Mpc}^{-3}$. The prediction for Compton-thick AGN based on the synthesis model of X-ray background in Gilli et al. (2007) is consistent with this value.
\end{abstract}

Key words. infrared: galaxies - X-rays: galaxies - galaxies: active

\section{Introduction}

A complete knowledge of the local active galactic nuclei (AGN) demography (i.e. their census and physical properties) is the essential starting point to be able to study the AGN evolution at cosmological distances. Indeed all models developed so far to address the problem of birth and growth of super massive black holes (SMBHs) in galaxies are forced to reproduce many observational constraints among which the correct mass and number of AGN observed locally (Marconi et al. 2004). While unobscured AGN can be easily detected and studied both in the optical band and in X-rays, the detection of absorbed AGN becomes more and more difficult as the amount of circum-nuclear obscuring medium intercepted along the line of sight increases. This is particularly true for heavily obscured sources (intrinsic column density, $N_{\mathrm{H}}>5 \times 10^{23} \mathrm{~cm}^{-2}$ ) and even more for Compton-thick AGN $\left(N_{\mathrm{H}}>10^{24} \mathrm{~cm}^{-2}\right)$ that are predicted to constitute more than half of the total number of AGN (Gilli et al. 2007). While for less obscured AGN the X-ray photons above few $\mathrm{keV}$ can penetrate the torus making the source nucleus, at least partially, directly visible to the observer and the column density and luminosity measurable, for Compton-thick AGN the primary radiation is almost completely absorbed in the X-rays. For these sources, the spectrum below $10 \mathrm{keV}$, is dominated by the so called Compton reflection/scattering component (e.g. continuum emission reflected by the torus) which is more than an order of magnitude fainter with respect to the direct component. Moreover, in spite of the different values of intrinsic $N_{\mathrm{H}}$, the shape of Compton-thin and Compton-thick AGN spectra below $10 \mathrm{keV}$ could be very similar. Indeed, if the statistics is not really good enough, this part of the spectrum could be usually well fitted by an absorbed $\left(N_{\mathrm{H}} \sim 5 \times 10^{23} \mathrm{~cm}^{-2}\right)$ transmitted component or by a Compton reflection component (see e.g. Maiolino et al. 1998; Braito et al. 2004). Because the reflection component has a broad Compton reflection hump in the 15-100 keV continuum, harder data are important to complement lower energies data and to investigate the nature of the sources (e.g. Severgnini et al. 2011; Trippe et al. 2011).

Even if the absorption is less severe above $10 \mathrm{keV}$, nonetheless even harder X-ray surveys could be strongly biased against the selection of Compton-thick AGN due to the Compton downscattering effect (Matt et al. 1999; Malizia et al. 2009; Burlon et al. 2011). In particular, by using a complete sample of AGN detected by Swift-BAT in the first three years of the survey, Burlon et al. (2011) have shown and quantified these effects at energies higher than $15 \mathrm{keV}$ for mildly $\left(N_{\mathrm{H}}\right.$ of the order of a few times $\left.10^{24} \mathrm{~cm}^{-2}\right)$ and heavily $\left(N_{\mathrm{H}} \geq 10^{25} \mathrm{~cm}^{-2}\right)$ Compton-thick AGN. They estimate that for a mildly Compton-thick AGN only $50 \%$ of the nuclear trasmitted flux is visible above $15 \mathrm{keV}$ and this fraction becomes only a few percent for heavily Comptonthick AGN. Therefore, even using hard X-ray data, Compton thick sources are very difficult to detect and the computation of their volume density requires significant corrections.

An alternative wavelength for the selection of heavily obscured AGN is the mid-infrared (mid-IR) band (see e.g. Georgantopoulos et al. 2011, and references therein), where the optical and UV photons of the primary source are re-emitted after having been reprocessed by hot dust. Since 
this band is less affected by obscuration than optical band and X-rays, AGN selection at these wavelengths is less biased against obscured AGN. However, AGNs usually represent only a small fraction of all sources detected in IR surveys compared to the far more numerous IR emitters, such as Galactic sources and normal and starburst galaxies. For this reason, to efficiently select AGN, it is convenient to complement the IR band with $\mathrm{X}$-ray data, where the galaxy and star contribution is minimal. By comparing 2-10 keV and IR fluxes it is possible to distinguish unobscured from obscured sources being the first ones relatively unbiased with respect to the extinction, while the second ones strongly depressed as the $N_{\mathrm{H}}$ value increases.

In this paper we present a well defined sample of Comptonthick AGN selected in the local Universe by combining mid-IR (IRAS) and X-ray (XMM-Newton) data. The method/diagram used to select the sample is discussed in Sect. 2 and the sample is presented in Sect. 3. We discuss the efficiency and the completeness of the method. We derive the Compton-thick AGN surface density in Sect. 4 and their space density in Sect. 5 where we also compare our results with those found in the literature. Summary and conclusions are presented in Sect. 6.

\section{Diagnostic plot}

As already mentioned, one way to select heavily obscured AGN candidates and to distinguish them from less obscured sources is to compare the X-ray emission below $10 \mathrm{keV}$ (strongly depressed by the absorption in Compton-thick AGN) with the emission from other bands less affected by the absorption, like harder X-rays or mid-IR $(12-25 \mu \mathrm{m})$ band (produced by the presence of large amounts of dust absorbing, thermalizing and re-emitting the optical and UV photons of the primary source). While hard X-rays can be strongly affected by the Compton down scattering effect, mid-IR selection appears to be relatively unbiased with respect to extinction even in the case of Comptonthick sources (Brightman \& Nandra 2011; Horst et al. 2008).

Starting from this consideration, we propose here a new diagnostic plot to select Compton-thick AGN in the local Universe. This plot is based on the combination of the ratio between the $2-12 \mathrm{keV}(F(2-12 \mathrm{keV}))$ and the mid-IR ( $F$ (mid-IR)) flux with the XMM-Newton colors (hardness ratio HR). We expect that Compton-thick sources are characterized by a lower $F(2-12 \mathrm{keV}) / F$ (mid-IR) ratio with respect to less obscured AGN (see e.g. Polletta et al. 2006; Severgnini et al. 2008). Since starburst galaxies are characterized by similarly low values of $F(2-12 \mathrm{keV}) / F$ (mid-IR) ratio, we propose here to use the X-ray colors to separate star-forming galaxies from Compton-thick AGN. While obscured AGN are characterized by hard X-ray emission, the soft emission due to star-formation activity will produce lower HR values (i.e. HR $<-0.1$, see Della Ceca et al. 2004) with respect to that of obscured AGN.

As a first step we have plotted the X-ray and mid-IR information for different samples of X-ray sources for which the nature has been already studied in the literature (i.e. unabsorbed and absorbed Compton-thin AGN; Compton-thick AGN and star-forming galaxies). The diagram is shown in Fig. 1 where the $F(2-12 \mathrm{keV}) /\left(v_{25} F_{25}\right)$ is plotted as a function of $\mathrm{HR} 4{ }^{1}$. We use this figure to define the region where looking for Compton-thick AGN: $F(2-12 \mathrm{keV}) /\left(v_{25} F_{25}\right)<0.02$

\footnotetext{
1 HR4 is defined using the two following bands: $2-4.5 \mathrm{keV}$ and 4.5-12 keV: HR4 $=\frac{\operatorname{CTS}(4.5-12 \mathrm{keV})-\mathrm{CTS}(2-4.5 \mathrm{keV})}{\operatorname{CTS}(4.5-12 \mathrm{keV})+C T S(2-4.5 \mathrm{keV})}$, where CTS are the vignetting corrected count rates in the energy ranges reported in bracket. See Watson et al. (2009) for details.
}

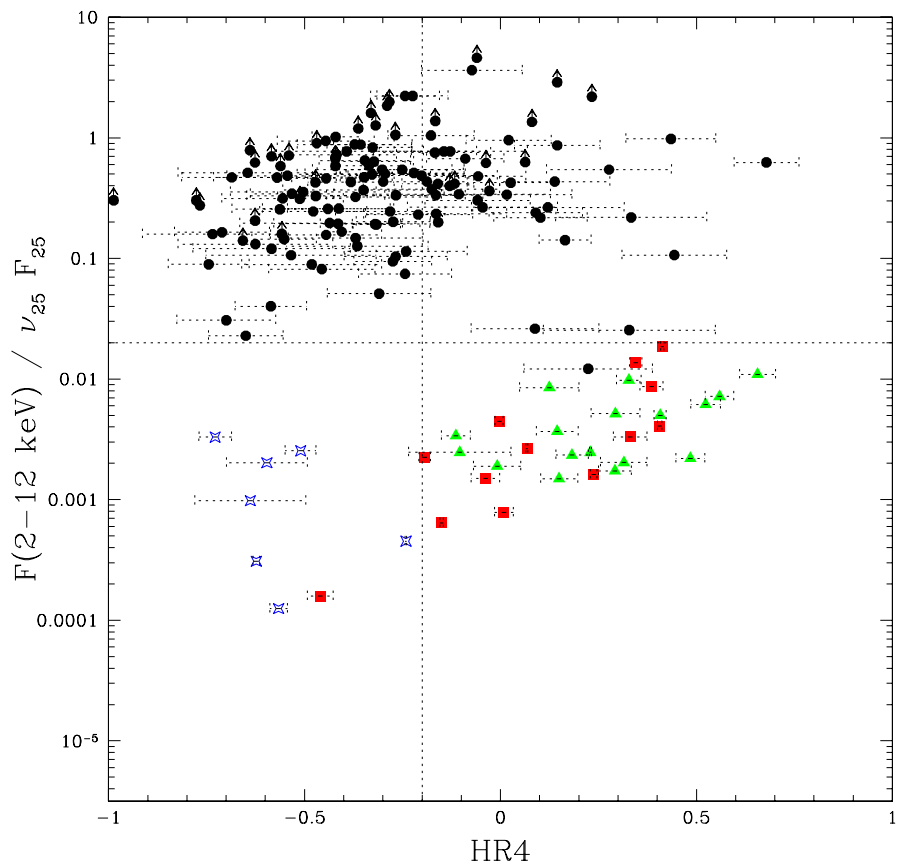

Fig. 1. $F(2-12 \mathrm{keV}) /\left(v_{25} F_{25}\right)$ vs. HR4 diagnostic plot. Filled circles (black symbol in the electronic version only) are unabsorbed and absorbed Compton-thin AGN $\left(N_{\mathrm{H}}<10^{24} \mathrm{~cm}^{-2}\right)$ taken from two different $\mathrm{X}$-ray samples in the literature (XMM-HBS sample - Della Ceca et al. 2008b; XMDS survey - Tajer et al. 2007; Polletta et al. 2007). Stars (blue objects in the electronic version only) are a sample of local starburst galaxies (Ranalli et al. 2003) and squares (red objects in the electronic version only) and triangles (green objects in the electronic version only) are local "confirmed" and "candidate" Compton-thick AGN, respectively, taken by the compilation of Della Ceca et al. (2008a).

and HR4 $>-0.2$. Filled black circles (131 objects) are all the sources with mid-IR information ${ }^{2}$ belonging to two X-ray different surveys: the XMM-Hard Bright Sample (XMM-HBS, Della Ceca et al. 2008b; Caccianiga et al. 2004; Severgnini et al. 2008) and the XMDS survey (Tajer et al. 2007; Polletta et al. 2007). $\mathrm{X}$-ray information have been taken from the 2XMM-slim catalogue (Watson et al. 2009). The XMM-HBS sources plotted in Fig. 1 are sources for which we obtained Spitzer proprietary data (cycle-3, P.I. Severgnini); they have a redshift range of $0.1<z<0.7$. The XMDS sources are mainly at $z<1.5$ with some sources up to $z=3.5$ (see redshift distribution in Tajer et al. 2007). All but one (the filled circle in the bottom part of the panel, $F(2-12 \mathrm{keV}) /\left(v_{25} F_{25}\right)=0.012$, HR4 $\left.=0.23\right)$ have $F(2-12 \mathrm{keV}) /\left(v_{25} F_{25}\right)>0.02$ (see Fig. 1) and for all of them there is no evidence for the presence of a Compton-thick AGN (see the relevant papers). The only source in which a Comptonthick AGN could be present is the filled circle in the bottom part of the panel, see Polletta et al. (2007). Stars (7 sources, blue objects in the electronic version only) are local optically selected star-forming galaxies taken from the sample of Ranalli et al. (2003). We have considered only those sources without evidence of a possible AGN. Finally, squares (13 sources, red objects in the electronic version only) and triangles (17 sources, green objects in the electronic version only) are local $(z<0.05)$ "confirmed" and "candidate" Compton-thick AGN, respectively, taken from the compilation of Della Ceca et al. (2008a). The so

2 For these sources, $24 \mu \mathrm{m}$ Spitzer/MIPS data have been used. In order to adopt an uniform notation for all the sources in the paper, we report in Fig. 1 the $25 \mu \mathrm{m}$ fluxes, assuming a negligible correction to go from 24 to $25 \mu \mathrm{m}$ flux in $v F_{v}$. 
called "confirmed" Compton-thick have been identified thanks to observations above $10 \mathrm{keV}$ with BeppoSAX, INTEGRAL, Swift/BAT and Suzaku, while the "candidate" Compton-thick AGN are sources with observations only below $10 \mathrm{keV}$. Both for star-forming and for Compton-thick AGN we have considered only those sources present in the 2XMM-slim catalogue (Watson et al. 2009) and with an IRAS detection. All but one (NGC 3690, see Sect. 3.2) of the local Compton-thick AGN plotted in Fig. 1 are placed in the lower-right part of the diagram.

Even if the comparison of different samples, selected in different ways and with different redshifts, is not indicative of the real efficiency and completeness of the proposed method, at first glance, it suggests that this diagram could be actually reliable in selecting local Compton-thick AGN.

In the next section we will test the efficiency of the proposed method and we will investigate if this diagram can provide a well defined and complete sample of local Compton-thick AGN from which it is possible to derive their surface and space density.

\section{The sample of Compton-thick AGN candidates}

To build up a new sample of Compton-thick candidates using the diagram shown in Fig. 1, we have crosscorrelated the IRAS Point Source Catalog (PSC, 245889 sources, see http://irsa.ipac.caltech.edu/IRASdocs/ exp.sup/index.html for details) at $25 \mu \mathrm{m}$ (we exclude sources with a $25 \mu \mathrm{m}$ flux density quality flag equal to 1 , corresponding to upper limit, see Helou \& Walker 1988) with the incremental version of the v1.0 2XMM slim catalogue that contains 221012 sources. We consider only sources with $F(4.5-12 \mathrm{keV})>10^{-13} \mathrm{erg} \mathrm{cm}^{-2} \mathrm{~s}^{-1}$ and likelihood parameter $>12$ in the $0.2-12 \mathrm{keV}$ band in order to maximize the number of counts for each source and to perform a reliable spectral analysis. To minimize the possible contamination of Galactic sources, we select only those sources having a high Galactic latitude $\left(\left|b^{\mathrm{II}}\right|>20^{\circ}\right)$. We have used a matching radius of 15" (see http://heasarc.gsfc.nasa.gov/W3Browse/ iras/iraspsc.html) and as a second step we have excluded all the sources having an X-ray counterpart more than $10^{\prime \prime}$ (see Watson et al. 2009) away from the optical source associated to the infrared emission reported in the PSC catalogue. By repeating several times the same correlation by shifting in declination one of the two catalogues of several degrees, we find that the number of spurious sources is negligible $(<1)$. The final list contains $145 \operatorname{IRAS}(25 \mu \mathrm{m})-2 \mathrm{XMM}$ matches with a mid-IR flux at $25 \mu \mathrm{m}$ ranging from 0.14 to $544 \mathrm{Jy}$.

As discussed in the previous section, on the basis of the plot reported in Fig. 1 we define as heavily obscured AGN region the zone with $F \mathrm{x} /\left(v_{\mathrm{IR}} F_{\mathrm{IR}}\right)<0.02$ and $\mathrm{HR} 4>-0.2$ (i.e. the lower-right region). By plotting the results of the IRAS-2XMM cross-correlation on the $F(2-12 \mathrm{keV}) /\left(v_{25} F_{25}\right)$-HR 4 plane we find 44 sources in the region associated to heavily obscured AGN (see Fig. 2, filled squares, red symbols in the electronic version only), 43 of which are extragalactic sources (the only Galactic object is the isolated encircled source in the bottom right part of the diagram). For all sources, the redshift is already reported in the literature (see Table 1). The redshift distribution is shown in Fig. 3. The full sample is at $z<0.1$ and $98 \%$ of the sources have $z<0.07$.

\subsection{X-ray properties of the Compton-thick candidates}

As a first step, we checked in the literature if an X-ray classification exists for all the sources in Table 1 . We find that a

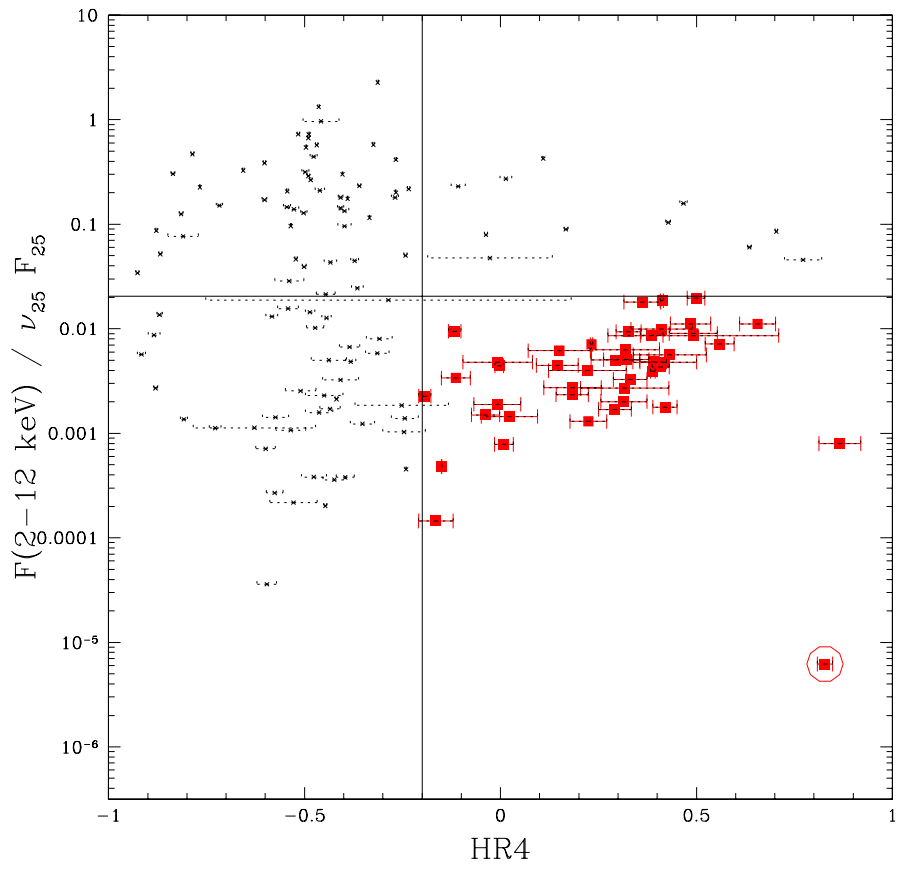

Fig. 2. $F(2-12 \mathrm{keV}) /\left(v_{25} F_{25}\right)$ vs. HR4 diagnostic plot for the 145 sources found by cross-correlating the PSC IRAS catalogue at $25 \mu \mathrm{m}$ and the $2 \mathrm{XMM}$ catalogue. Filled squares (red symbols in the electronic version only) are the 44 sources that have flux ratios and X-ray colors typical of Compton-thick AGN. The isolated object in the bottom-right part of the diagram marked with an empty circle is the only Galactic source $\left(\mathrm{V}^{*} \mathrm{R}\right.$ Aqr) present in the Compton-thick candidate region.

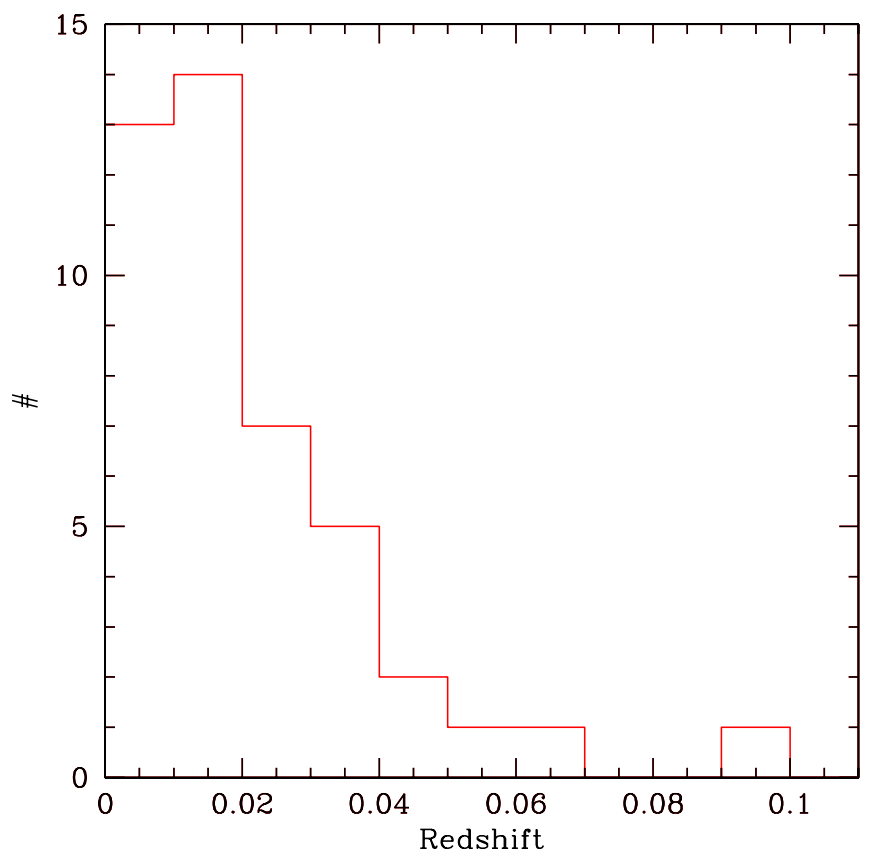

Fig. 3. Redshift distribution of the 43 extragalactic sources that lie in the Compton-thick region of the plot reported in Fig. 2.

large fraction of them (30/43) is already known as Comptonthick AGN on the basis of a direct measure of the absorption cut-off or through indirect arguments, such as the presence of a strong Iron emission line at $6.4 \mathrm{keV}$. Twenty Compton-thick belong to the compilation of Della Ceca et al. (2008a) and they are plotted also in Fig. 1. For 13 Compton-thick AGN, 


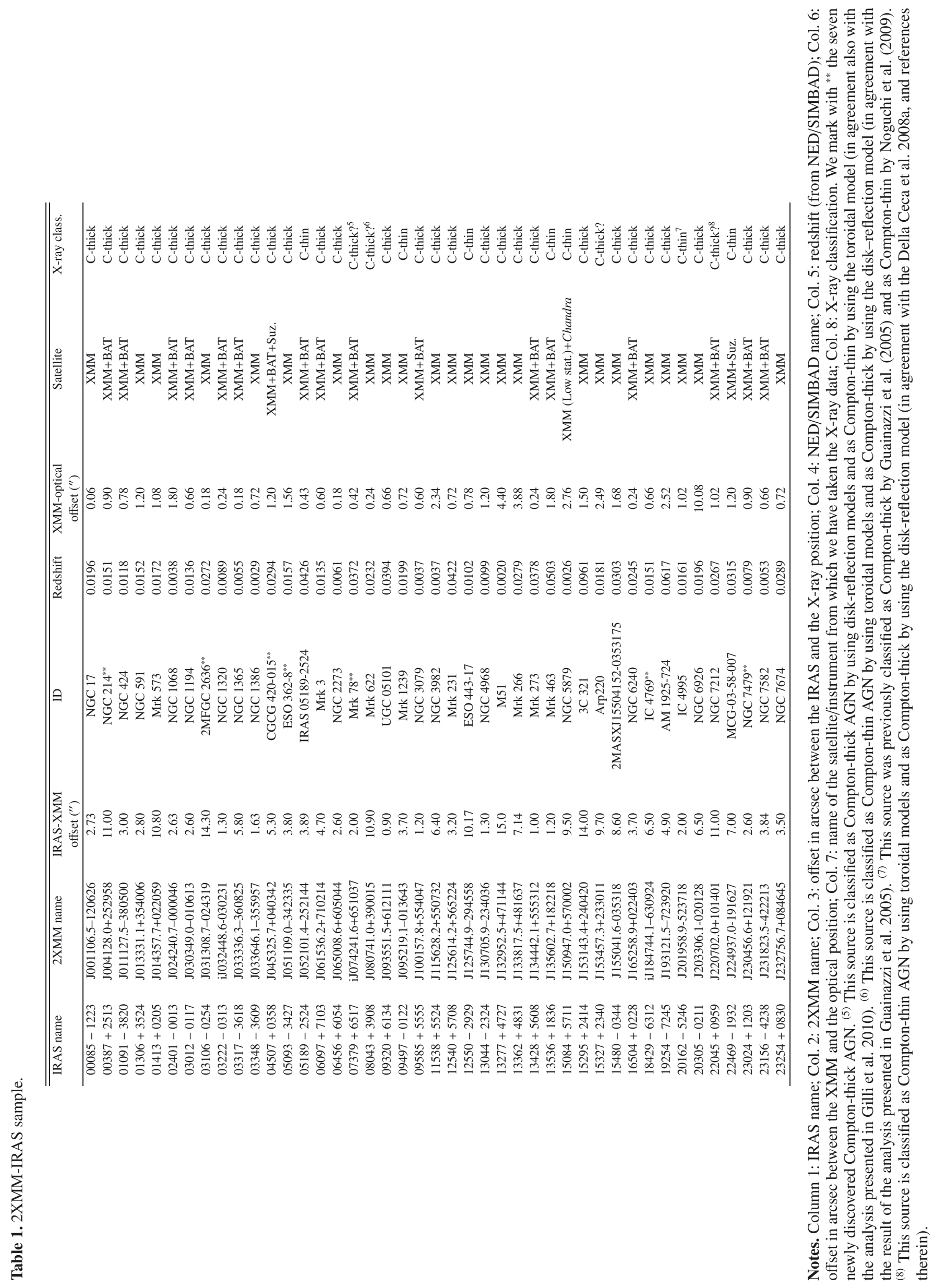


the classification has been confirmed also thanks to observations above $10 \mathrm{keV}$ (NGC 424, NGC 1068, Mrk 3, NGC 3079, Mrk 231, M51, NGC 6240, NGC 7674, see Della Ceca et al. 2008a - Mrk 273; see Teng et al. 2009 - NGC 2273; see Awaki et al. 2009-NGC 7582; see Bianchi et al. 2009-NGC 1365; see Risaliti et al. 2005 - AM 1925-724; see Braito et al. 2009). Three of them (NGC 1365, NGC 7674 and NGC 7582) show rapid Compton-thick/Compton-thin transitions and they are known as "changing-look" AGN. Finally, one source is Arp 220, that has been extensively studied so far in several bands. Many of the features detected in the X-ray spectrum (a flat continuum - Ptak et al. 2003 - and a prominent $\mathrm{Fe} \mathrm{K} \alpha$ emission line, $E W \sim 1.9 \mathrm{keV}$ - Iwasawa et al. 2005) suggest the presence of a heavily obscured AGN. This hypothesis is also the most favorite one after the analysis of the Suzaku data by Teng et al. (2009). Thus we consider this object as a possible Compton-thick AGN.

In order to obtain a uniform analysis for all of the Comptonthick AGN candidates, we have performed our own spectral analysis using the XMM data for the 42 sources in the sample with more than 100 net counts in the $0.5-12 \mathrm{keV}$ range. For the remaining one (NGC 5879) the statistics of the XMM data is not good enough (from 15 to 60 counts) to allow an appropriate $\mathrm{X}$-ray spectral analysis. Since good (>100 net counts) Chandra data are available for this latter object, we use them to study its $\mathrm{X}$-ray spectral properties. We have applied both disk reflection models (i.e. pexrav model Magdziarz \& Zdziarski 1995) and the recent model proposed in the case of neutral toroidal X-ray reprocessor in AGNs (Murphy \& Yaqoob 2009). We inferred the possible presence of Compton-thick AGN mainly through the detection of the absorption cut-off or through indirect arguments, such as the presence of dominant $2-10 \mathrm{keV}$ reflection/scattering emission plus a prominent $(E W>400 \mathrm{eV})$ Iron line. To further investigate the nature of our sources we have obtained hard X-ray data from the catalogue obtained after 54 months of SwiftBAT observations (Cusumano et al. 2010) for 17 sources and we used this X-ray hard data to better constrain the absorbing column density. We also obtained Suzaku observations for two of them (IRAS 04507+0358 and MCG-03-58-007, $100 \mathrm{ks}$ each). A detailed description of the analysis done on the XMM data of the sources not known as Compton-thick from the literature, combined, in some cases, with Chandra, BAT and Suzaku data, will be reported in a companion paper (Severgnini et al., in prep.).

In the last two columns of Table 1 we report: the satellites/instruments from which we have taken the X-ray data and the X-ray classification. We classify a source as "Comptonthick" AGN (32 sources) if we obtain an indication of a column density $\left(N_{\mathrm{H}}\right)$ larger than $10^{24}$ with both the models used (disk-reflection and toroidal models), while we adopt the classification "Compton-thick?" for 3 sources for which the presence of a Compton-thick AGN is model dependent and in the case of Arp 220. Our X-ray analysis confirms the classification as Compton-thick AGN taken from the literature in all cases except for one source (IC 4995, see Guainazzi et al. 2005). In addition to these, we find 7 newly discovered Compton-thick AGN (marked with a double asterisk in Table 1). One of these is IRAS $04507+0358$, that we have extensively discussed in Severgnini et al. (2011).

\subsection{Efficiency and completeness of the method}

Efficiency - The diagnostic plot proposed here could be considered as an efficient way to select local Compton-thick sources in the nearby Universe. As reported in the previous section, for $\sim 84 \%$ of the sources populating the Compton-thick region the presence of a Compton-thick AGN is suggested or confirmed by the X-ray spectral analysis. For comparison, the efficiency in finding Compton-thick AGN using other X-rayto-mid-infrared diagnostic ratio (e.g. $L_{\mathrm{X}} / L_{6 \mu \mathrm{m}}$, as recently proposed by Georgantopoulos et al. 2011) is $50 \%$ and in a hard X-ray survey, like that presented in Burlon et al. (2011) or in the recently published all-sky sample of AGN detected by BAT in 60 months of exposure (Ajello et al. 2012), is about 5-6\%.

Completeness - While the samples reported in Fig. 1 can not be used to estimate the efficiency of the proposed method, we can use them to state, at first glance, its completeness. Indeed, even if the Compton-thick sample does not include all the Compton-thick AGN known so far, the sources plotted in Fig. 1 have been not chosen on the basis of their X-ray-to-IR ratio or on the basis of their X-ray colors. In this sense, they can be considered representative of the AGN Compton-thick population.

As already discussed in Sect. 2, there is just 1 source, NGC 3690, in the Compton-thick compilation reported by Della Ceca et al. (2008a) that falls outside the Compton-thick region considered here. We discuss it in more details in the following.

NGC 3690 falls in the lower-left part of the plot (i.e. the star-forming region). It is one of the two merging galaxies of the LIRG Arp 299 (Sanders et al. 2003; Heckman et al. 1999; Della Ceca et al. 2002; Ballo et al. 2004). The optical spectroscopic classification puts this source at borderline between starburst and LINER (Coziol et al. 1998), while the X-ray analysis clearly reveals the presence of a strongly absorbed AGN in the system (Della Ceca et al. 2002; Ballo et al. 2004). The 2-10 keV continuum is due to a combination of reprocessed AGN emission (reflection and/or scattering) and starburst activity which, most probably, dominates and produces the soft HR4 (=-0.396) observed. This is the only source already known as Compton-thick AGN which lies in the star-forming region of both Figs. 1 and 2. As a further check of the possible presence of Compton-thick AGN in this part of the plot, we have verified how many sources of Fig. 2 placed in this part have a detection in the hard X-rays. To this end we considered the 54-months Swift-BAT catalogue by Cusumano et al. (2010). The only source with hard X-ray detection is M 82, which is considered one of the prototype of starburst galaxies (Sakai \& Madore 1999). The hard emission detected in this source is most probably due to the presence of a Ultra-luminous compact X-ray source (X-1, Miyawaki et al. $2009)$ with a bolometric luminosity of $(1.5-3) \times 10^{40} \mathrm{erg} \mathrm{s}^{-1}$. No evidence of Compton-thick AGN in this object and no evidence of Compton-thick AGN in the other sources populating the star-forming region of the plot can be derived by hard X-ray observations. This part of the plot is populated by star-forming galaxies or low-luminosity Seyfert/LINERs in which the X-ray emission is most probably dominated by star-forming activity.

By taking into account that we are considering only those sources with IRAS PSC and XMM-Newton information and with $F_{25}>0.5 \mathrm{Jy},\left|b^{\mathrm{II}}\right|>20^{\circ}$ and $F(4.5-12 \mathrm{keV})>$ $10^{-13} \mathrm{erg} \mathrm{cm}^{-2} \mathrm{~s}^{-1}$, there are 20 Compton-thick AGN in the compilation of Della Ceca et al. (2008a) that satisfy these criteria. Since, as quoted above, our Compton-thick selection miss 1 of them, we state that, in a first approximation, our method is complete at $95 \%(C \sim 0.95)$.

\section{Compton-thick AGN surface density}

We now want to use the selected sample to estimate the number of Compton-thick AGN in the local Universe $(z<0.1)$. As 
discussed above, mid-IR band is less affected by the absorption and, therefore, the selection function is expected to be relatively flat (see also Brightman \& Nandra 2011).

Since the IRAS survey is complete down to $\sim 0.5 \mathrm{Jy}$ at $25 \mu \mathrm{m}$ (Helou \& Walker 1988), hereafter we will refer to this flux limit to derive statistical considerations on Compton-thick AGN. Out of the 43 sources in the Compton-thick box, 34 have $F_{25} \geq 0.5 \mathrm{Jy}$. Twenty-six are classified as "Compton-thick" and 3 as "Compton-thick?". Thus we observe 26-29 Compton-thick AGN down to a flux limit of $F_{25}=0.5 \mathrm{Jy}$.

In order to derive the density of Compton-thick we have to take into account three problems that affect the sample discussed here.

First, we search for local Compton-thick AGN by considering only those sources with $F(2-12 \mathrm{keV}) /\left(v_{25} F_{25}\right)<0.02$ and HR $4>-0.2$. We have already discussed the completeness $C$ of our selection method in Sect. 3.2.

Second, the effective area of sky covered by our sample is not known a-priori. The problem is connected to the $2 X M M$-Newton catalogue which includes both sources falling serendipitously in the field-of-view of the telescope and the targets of the pointings. Considering only the serendipitous sources, the sky area covered by the $2 X M M-N e w t o n$ catalogue is relatively small ( $\sim 360 \mathrm{deg}^{2}$, Watson et al. 2009). Based on previous estimate of the surface density of Compton-thick AGN, the expected number of nearby Compton-thick AGN falling by chance in this area is negligible $(<1$, see e.g. Burlon et al. 2011) so our sample is made almost exclusively by sources that have been targeted by the XMM-Newton telescope (all but 2 sources are targets). Therefore, the probability of finding a source in the $2 X M M$ catalogue, is not anymore connected to the real area covered by the catalogue but it depends on how frequently that type of astrophysical source has been observed. Ideally, if all or nearly all the sources under study with a flux above a given flux limit have been pointed by XMM-Newton, the covered area can be considered equal to the entire sky. If, on the contrary, only a fraction of sources have been pointed, the effective area must be scaled down proportionally. We call this fraction $F_{\text {target }}$. Since the pointed sources do not constitute a representative sample, the value of $F_{\text {target }}$ is expected to be different for different classes of astrophysical sources.

Third, our sample is flux limited in two different bands, i.e. the $25 \mu \mathrm{m}$ and the X-ray bands, so it cannot be considered as a purely mid-IR selected sample. For a given mid-IR flux limit, the effect of the X-ray limit is to exclude a number of sources. We refer as $F_{\mathrm{Xl}}$ the fraction of objects that pass the X-ray limit (i.e. $F_{\mathrm{Xl}}=1$ if the $\mathrm{X}$-ray limit is not important).

If all the three factors discussed above $\left(C, F_{\text {target }}\right.$ and $\left.F_{\mathrm{Xl}}\right)$ are estimated, we can infer the number of Compton-thick AGN $\left(N_{\mathrm{CT}}\right)$ at the IRAS flux limit starting from the computed number of Compton-thick present in the sample and the relevant surface density:

$$
\begin{array}{r}
N_{\mathrm{CT}}\left(F_{25}>F_{\mathrm{LIM}}\right)=\frac{N_{\text {observedCT }}}{C \times F_{\mathrm{Xl}} \times F_{\text {target }}} \\
\rho^{\mathrm{CT}}\left(F_{25}>F_{\mathrm{LIM}}\right)=\frac{N_{\mathrm{CT}}}{A_{20}} \mathrm{src} \mathrm{deg}^{-2}
\end{array}
$$

where $A_{20}$ is the total sky area at high Galactic latitude $\left(\left|b^{\mathrm{II}}\right|>\right.$ $20 \mathrm{deg}$ ) and $F_{\text {LIM }}$ is the flux limit at $25 \mu \mathrm{m}$.

In the following, we present different methods to estimate the two fractions, $F_{\text {target }}$ and $F_{\mathrm{Xl}}$.

\subsection{Estimate of $F_{\text {target }}$}

As explained above, the sample of Compton-thick analyzed in this paper is made mainly by sources that have been chosen as targets of XMM-Newton telescope. In order to quantify the value of $F_{\text {target }}$, i.e. the fraction of sources that have been pointed by $X M M-N e w t o n$, we have analyzed two samples of sources that are in many aspects similar to the one considered here. The first one is the sample of Seyfert2/CT AGN discovered in the Swift-BAT survey (Burlon et al. 2011) while the second one is the sample of Seyfert 2 included in the extended $12 \mu \mathrm{m}$ sample (Rush et al. 1993). The first one is a complete, flux-limited sample of local AGN at $\left|b^{\mathrm{II}}\right|>15 \mathrm{deg}$ collected by the Swift-BAT instrument in the first three years of the survey, while the second one is a $12 \mu$ m flux-limited sample of 893 galaxies at $\left|b^{\mathrm{II}}\right|>25 \mathrm{deg}$ from the IRAS Faint Source Catalogue (Moshir 1991). Both samples are purely flux limited samples and, in both cases, the selection is not related to the (soft) X-ray properties of sources. Since the properties (IR fluxes, redshift) of these sources are very similar to the Compton-thick AGNs present in our sample (indeed, the overlap between these samples is large) it is reasonable to assume that the fraction of Seyfert 2 in Swift-BAT sample or in the "extended" $12 \mu \mathrm{m}$ sample that have been observed by XMM-Newton gives a rough approximation of the value of $F_{\text {target }}$. We have thus positionally cross-correlated these two catalogues with the $2 \mathrm{XMM}$ catalogue. We find that $50 \%$ of the $12 \mu \mathrm{m}$ classified as Seyfert 2 have been pointed with XMM-Newton. Since $12 \mu \mathrm{m}$ sample is not spectroscopically complete (Hunt \& Malkan 1999; Brightman et al. 2011) and since the optical elusiveness of X-ray selected AGN is a well known critical problem (see e.g. Caccianiga et al. 2007; Severgnini et al. 2003; Maiolino et al. 2003, and references therein) we have estimated the fraction of XMM-Newton targets including also the sources classified as LINERS or "high far infrared" sources (that potentially may contain an hidden Compton-thick AGN). We find that the fraction decreases to $40 \%$.

Finally, if we consider only the AGN in the Swift-BAT sample with a measured $N_{\mathrm{H}}$ larger than $10^{24} \mathrm{~cm}^{-2}$ we find a somewhat higher fraction (63\%), although considering the small numbers ( 7 out of 11 sources observed with XMM-Newton), this fraction is fully consistent with the one found considering all the Sy2s. We conclude that a reliable estimate of $F_{\text {target }}$ is $0.5 \pm 0.1$.

\subsection{Estimate of $F_{X I}$}

The value of $F_{\text {target }}$ computed above does not take into account the fact that we are considering only the sources with an X-ray flux above $10^{-13} \mathrm{erg} \mathrm{cm}^{-2} \mathrm{~s}^{-1}$. The presence of this limit, which has been set in order to make the X-ray spectral analysis more reliable, exclude from our sample a number of Compton-thick AGNs. We evaluate the fraction of missing sources by re-running the positional cross-correlation between the 2XMM and the IRAS catalogues without imposing any limit on the X-ray flux. After the exclusion of Galactic sources and of ULX in nearby galaxies we find 53 sources in the "Compton-thick box" down to $F_{25}=0.5 \mathrm{Jy}$. At fainter X-ray fluxes the number of expected spurious matches (negligible in the original sample) probably could be important. Therefore, we have repeated several times the same correlation by shifting in declination one of the two catalogues of several degrees in order to get an estimate of the fraction of spurious sources.

We estimate a fraction of spurious matches of the order of $10 \%$ so the actual number of matches is about 48 , i.e. 14 more sources with respect to the original sample (34 sources, 
including non Compton-thick sources) down to the same $25 \mu \mathrm{m}$ flux in the Compton-thick box. We therefore estimate a value of $F_{\mathrm{Xl}}$ of $34 / 48 \sim 0.7$. We note that, following this method to compute the $F_{\mathrm{Xl}}$, we only consider the X-ray sources that are present in the $2 \mathrm{XMM}$ catalogue. Therefore, sources fainter than the $2 \mathrm{XMM}$ flux limit are not included in this computation. It could be argued that in this way the fraction of sources missed because of the X-ray limit is underestimated. This would be true is we were considering only serendipitous source. We recall, however, that we are dealing with sources that are targets of the $X M M-N e w t o n$ observation. If a source has been pointed, then it is usually detected ${ }^{3}$ and, therefore, present in the $2 X M M$ catalogue. On the contrary, if a source is not in the 2XMM catalogue, this means that it has not been chosen as a target. Therefore, the problem of the sources that are not included in the 2XMM catalogue is already accounted for during the $F_{\text {target }}$ step and it does not require any further correction.

\subsection{The density of Compton-thick AGN}

Using the values of $C, F_{\text {target }}$ and $F_{\mathrm{Xl}}$ derived above and the number of Compton-thick AGN found in our sample (26-29) down to a flux limit of $0.5 \mathrm{Jy}$ at $25 \mu \mathrm{m}$, we can compute the number of Compton-thick AGN and their surface density. We find:

$N_{\mathrm{CT}}\left(F_{25}>0.5 \mathrm{Jy}\right) \sim 83 \pm 5$

$\rho^{\mathrm{CT}}\left(F_{25}>0.5 \mathrm{Jy}\right) \sim 3 \times 10^{-3} \mathrm{src} \mathrm{deg}^{-2}$.

\section{Comparison with other samples}

As discussed in the previous sections, to detect and study Compton-thick AGN is not easy, even in the local Universe. Often, the low X-ray statistics or the very high column density $\left(N_{\mathrm{H}}>5 \times 10^{24} \mathrm{~cm}^{-2}\right)$ prevent us from deriving the amount of absorption along the line of sight by using observations below $10 \mathrm{keV}$. For these sources, even at $E>10 \mathrm{keV}$ there is a strong bias against the detection of very obscured sources, as recently demonstrated by Burlon et al. (2011). In particular, these authors analyzed a complete sample of AGN detected by Swift-BAT in the first three years of the survey. They estimate the bias of the BAT instrument against the detection of Compton-thick AGN and they found that the real fraction of AGN with $N_{\mathrm{H}}$ ranging from $10^{24}$ to $10^{25} \mathrm{~cm}^{-2}$ should be a factor of 3-4 greater than the observed one, for a total of $\sim 40$ expected Compton-thick AGN down to a flux limit of $\sim 10^{-11} \mathrm{erg} \mathrm{cm}^{-2} \mathrm{~s}^{-1}$ and $\left|b^{\mathrm{II}}\right|>15^{\circ}$.

It is now interesting to compare the results obtained here with those reported in Burlon et al. (2011) or in the recent updated BAT all-sky catalogue published by Ajello et al. (2012). Given the different selection band (IR and hard X-rays respectively) we can compare the two surveys only by assuming an average hard X-ray-to-IR flux ratio typical for AGN. This ratio must be intrinsic, i.e. it should not include the effect of Compton-down scattering that reduces the hard X-ray flux. On the basis of the Unified model of AGN (Antonucci 1993), the average intrinsic X-ray-to-IR flux ratio can be simply

\footnotetext{
3 We have verified that the Sy 2 pointed by XMM have been actually detected. To do this, we have considered the 44 AGNs classified as "Seyfert type 2" in the XMM-Newton Master Log \& Public Archive and we have checked whether they appear also in the 2XMM catalogue of sources. We have found that 40 out of 44 objects are indeed present in the $2 \mathrm{XMM}$ catalogue and, therefore, they are detected. In the remaining 4 cases the source is not present in the 2XMM catalogue simply because the image has not been used to produce the $2 \mathrm{XMM}$ catalogue.
}

computed using the type 1 AGNs present in the BAT survey of Burlon et al. (2011), since the Compton-down scattering is completely negligible at the column densities observed in this type of sources. We measure an average $F_{(15-55 \mathrm{keV})} / F_{25}$ ratio of $\sim 5 \times 10^{-11} \mathrm{erg} \mathrm{s}^{-1} \mathrm{~cm}^{-2} \mathrm{Jy}^{-1}$ which implies that the $F_{25} \geq$ $0.5 \mathrm{Jy}$ of our surveys corresponds to an hard X-ray limit of $F_{(15-55 \mathrm{keV})} \sim 2.5 \times 10^{-11} \mathrm{erg} \mathrm{cm}^{-2} \mathrm{~s}^{-1}$. Using the BAT survey, we estimate that at this flux limit the density of Comptonthick sources, corrected for the Compton-down scattering ${ }^{4}$, is $7 \times 10^{-4} \mathrm{src} \mathrm{deg}^{-2}$ and $8 \times 10^{-4} \mathrm{src} \mathrm{deg}^{-2}$ from Burlon et al. (2011) and Ajello et al. (2012), respectively. These values are a factor $\sim 4$ below the density computed in our survey (see Sect. 4.3).

The origin of this large discrepancy could be related to the contamination of the observed IR flux from non-AGN activity, like the one, for instance, due to an intense star-formation. Indeed, a characteristic feature of the X-ray spectra of the Compton-thick sources in our sample is the almost ubiquitous presence of a thermal component that suggests the presence of star formation in the host galaxy. It is therefore possible that the observed $25 \mu \mathrm{m}$ flux is, at least in part, due to this extra "nonAGN" component. If this is the case, our sample includes AGN intrinsically fainter with respect to the Compton-thick AGN in the sample of Burlon et al. (2011).

As suggested by Fig. 1, the lower-left region in Fig. 2 should be dominated by star-forming activity. To evaluate the contribution of star-forming activity to the $25 \mu \mathrm{m}$ emission $\left(F_{25}(\mathrm{SF})\right)$ in addition to the AGN $\left(F_{25}(\mathrm{AGN})\right)$, we have considered all the sources populating this part of the diagram after excluding the sources classified as "Seyfert", "LINERs" or "Star" by NED". Using these sources we can thus estimate the mean $F_{25}(S F) / F_{(0.5-2 \mathrm{keV})}$ ratio of the star-forming galaxies (see Fig. 4) and use it to estimate the $F_{25}(S F)$ in the Compton-thick AGN. In particular, we use the $F_{(0.5-2 \mathrm{keV})}$ derived from our X-ray spectral analysis and by considering only the $0.5-2 \mathrm{keV}$ thermal component. We find that the host galaxies of our Compton-thick AGN have $25 \mu \mathrm{m}$ luminosities associated with the star-formation activity that ranges from about $6 \times 10^{8} L_{\odot}$ to $6 \times 10^{11} L_{\odot}\left(75 \%\right.$ of them have $\left.L_{25}<5 \times 10^{10} L_{\odot}\right)$, that are in good agreement with the typical IR luminosity range measured in local IRAS galaxies (see e.g. Rush et al. 1993). From the observed $F_{25}$ and the $F_{25}(S F)$ estimated from the soft X-ray flux we then obtain, by difference, the AGN contribution in all the Compton-thick sources. We find that, at the zeroth order, the mean AGN contribution to the total $25 \mu \mathrm{m}$ flux range from $40 \%$ to $10 \%$, i.e. in our sample the galaxy contribution at the IR band is not negligible.

We now consider only those objects in the original sample of 43 sources that have $F_{25}(\mathrm{AGN}) \geq 0.5 \mathrm{Jy}$. We find 20 sources, 15-16 of which are Compton-thick, i.e. the number of Comptonthick AGN is decreased of a factor $\sim 1.8$ (from 26-29 to $15-16$ ). This means that the Compton-thick AGN density at $F_{25}=$ $0.5 \mathrm{Jy}$, if only the AGN emission is considered, is $\sim(1.7 \pm 1) \times$ $10^{-3} \mathrm{src} \mathrm{deg}^{-2}$ a value that, considering the uncertainties on all the estimates, is compatible with the density estimated from the BAT survey (Burlon et al. 2011; Ajello et al. 2012). This confirms that Compton down scattering is important at hard X-ray energies and that the Compton-thick AGN densities estimated

\footnotetext{
4 We applied the same correction as estimated by Burlon et al. (2011) to remove the effect of the Compton-down scattering on the total number of Compton-thick AGN observed in the BAT survey.

5 NED (NASA/IPAC Extragalactic Database) is operated by the Jet Propulsion Laboratory, California Institute of Technology, under contract with the National Aeronautics and Space Administration.
} 


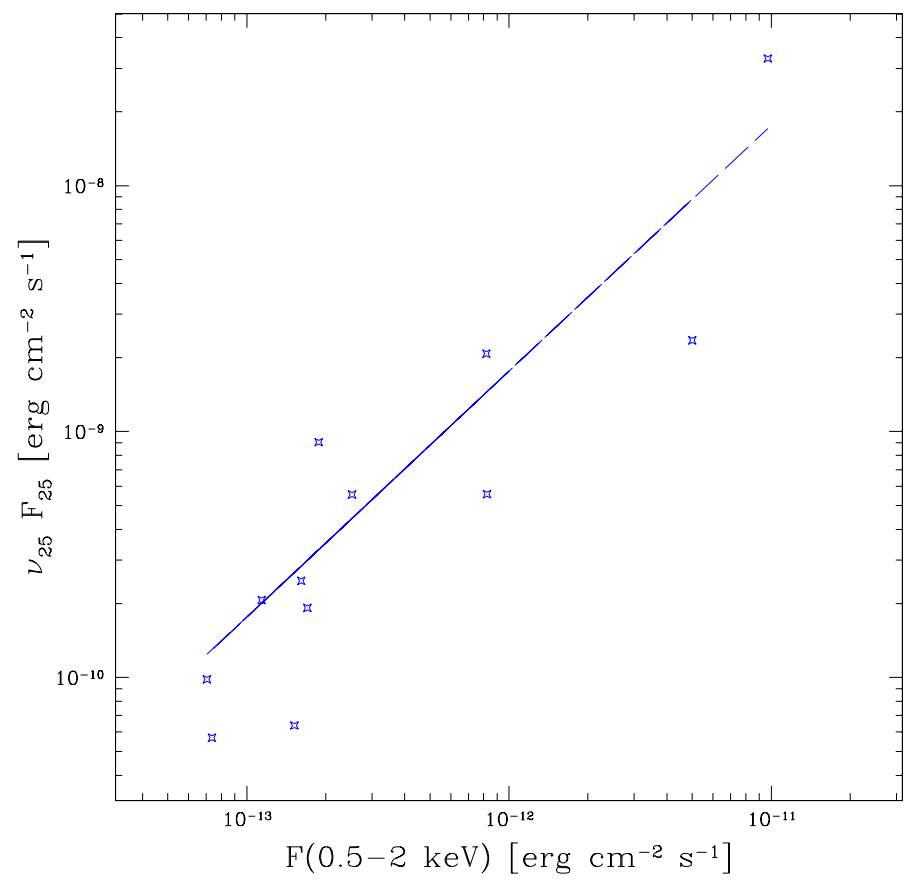

Fig. 4. Mid-IR $\left(v_{25} F_{25}\right)$ vs. X-ray $(F(0.5-2 \mathrm{keV}))$ fluxes of the sources populating the lower-left region in Fig. 2 after excluding the sources classified as "Seyfert", "LINERs" or "Star" by NED. The straight line (blue line in the electronic version only) indicates the mean value of the $v_{25} F_{25} / F(0.5-2 \mathrm{keV})$ of these sources.

from hard X-ray surveys must be significantly corrected, as done by Burlon et al. (2011). Although we have demonstrated that the infrared band is contaminated by star-forming emission, the corrections to apply in this case are lower with respect to those in the hard X-rays. An IR-based selection allows the discovery of the majority of the sources and, more importantly, is not biased (in principle) against high column densities because it is not affected by the Compton down scattering.

We finally estimate the co-moving space density of locally Compton-thick AGN. In order to allow a direct comparison with recent results obtained for higher redshift Compton-thick AGN, we estimated this density for Compton-thick AGN with $L_{X}>$ $10^{43} \mathrm{erg} \mathrm{s}^{-1}$.

Since we are dealing with an IR selected sample, we consider the AGN spectral energy distribution (SED) reported by Shang et al. (2011). These authors have compiled SED for 85 quasars using high-quality multi-wavelength data from radio to X-rays and they constructed the median SEDs for radio loud and radio quiet quasars. We derive the IR-to-X-ray luminosity ratio for AGN on the basis of their composite SED for radio quiet. We consider all the sources of our sample with $L_{25}>4 \times 10^{30} \mathrm{erg} \mathrm{cm}^{-2} \mathrm{~s}^{-1} \mathrm{~Hz}^{-1}$ (10 sources), that is the IR luminosity equivalent to $L_{(2-10 \mathrm{keV})}>10^{43} \mathrm{erg} \mathrm{s}^{-1}$. After rescaling the original sample for the different incompleteness discussed in Sect. 4, we estimated the co-moving space density of local $(0.004<z<0.06)$ Compton-thick AGN (with the $1 / V_{\max }$ method, Avni \& Bahcall 1980): $\Phi_{\mathrm{C}-\text { thick }} \sim\left(3.5_{-0.5}^{+4.5}\right) \times 10^{-6} \mathrm{Mpc}^{-3}$ (assuming $H_{0}=71, \Omega_{\lambda}=0.7$ and $\Omega_{\mathrm{M}}=0.3$ ).

In Fig. 5 we compare our estimate with the values measured by different authors at different redshifts (open and solid symbols) and with the predictions of the synthesis models of X-ray background (dashed lines). All the data and the models reported in Fig. 5 refer to sources with $L_{(2-10 \mathrm{keV})}>10^{43} \mathrm{erg} \mathrm{s}^{-1}$. Our value is in agreement with the co-moving space density obtained

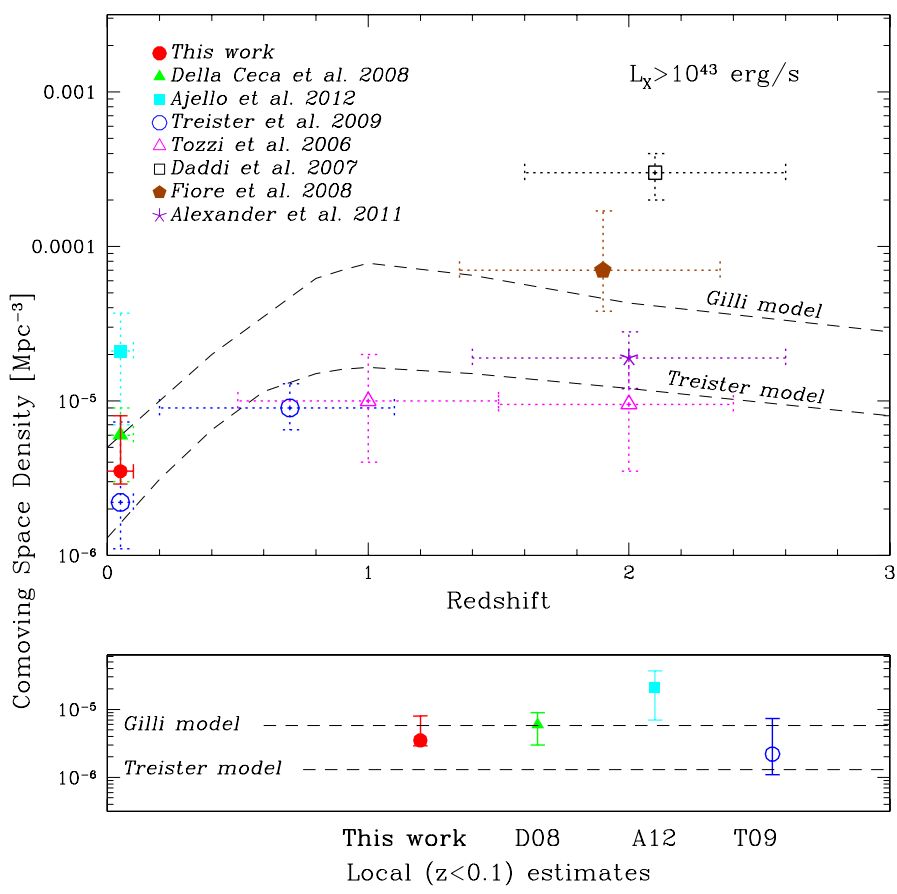

Fig. 5. Co-moving space density of Compton-thick AGN. All the data and the model plotted in the figure refer to $L_{\mathrm{X}}>10^{43} \mathrm{erg} \mathrm{s}^{-1}$. Filled circle (red symbol in the electronic version only) is the estimate obtained in this work, while the other local values are taken from Della Ceca et al. (2008b, solid triangle, green symbol in the electronic version), from Ajello et al. (2012, solid square, cyan symbol in the electronic version) and from Treister et al. (2009a, open circle at the local redshift, blue symbol in the electronic version only). As for higher redshift estimates: open circle (blue symbol in the electronic version only), filled pentagon (brown symbol in the electronic version only) and open square are the results obtained from the X-ray stacking analysis of undetected candidate Compton-thick AGN from Treister et al. (2009b), Fiore et al. (2008) and Daddi et al. (2007), respectively. The results obtained fron the X-ray spectral analysis from Tozzi et al. (2006) and Alexander et al. (2011) are marked with open triangles (magenta symbols in the electronic version only) and star (purple symbol in the electronic version only), respectively. The results are compared to the predictions of the models proposed by Gilli et al. (2007) and Treister et al. (2009a), dashed curves. The local co-moving space density estimates are reported also in the lower panel as a function of the different authors.

by integrating the X-ray luminosity function of Compton-thick AGN discussed in Della Ceca et al. $2008\left(\Phi_{\mathrm{C}-\text { thick }} \sim 6 \times\right.$ $10^{-6} \mathrm{Mpc}^{-3}$ adapted to $\left.H_{0}=71\right)$ and with the estimate reported by Treister et al. (2009a) for local Compton-thick AGN, while it is lower with respect to the value reported by Ajello et al. (2012). It is worth noting that Draper \& Ballantyne (2010) have corrected the local space density reported by Treister et al. (2009a) to account for the flux-luminosity relation for Comptonthick AGN described by Rigby et al. (2009); they found a value in very good agreement with our estimate $\left(\sim 3.6 \times 10^{-6} \mathrm{Mpc}^{-3}\right)$.

For completeness, we report in Fig. 5 also the different estimates of the co-moving space densities for higher redshift Compton-thick AGN ranging from $\Phi_{\mathrm{C}-\text { thick }} \sim 10^{-5} \mathrm{Mpc}^{-3}$ to $\Phi_{\mathrm{C}-\text { thick }} \sim 3 \times 10^{-4} \mathrm{Mpc}^{-3}$. We plot the results obtained from the $\mathrm{X}$-ray stacking analysis of undetected candidate Compton-thick AGN from Treister et al. (2009b), Fiore et al. (2008) and Daddi et al. (2007) and those obtained from the X-ray spectral analysis from Tozzi et al. (2006) and Alexander et al. (2011).

Finally, as for the comparison with the synthesis models of X-ray background, the result obtained by using the model of Gilli et al. (2007) is consistent, within the uncertainties, with the 
space density derived in this work, while the prediction obtained by the model presented in Treister et al. (2009a) is lower.

\section{Summary and conclusion}

We have presented a new method to select Compton-thick AGN in the local Universe, evaluated its efficiency and completeness.

The proposed method is based on the combination of the X-ray-to-mid-IR flux ratio $\left(F(2-10 \mathrm{keV}) /\left(v_{25} F_{25}\right)\right)$ with the X-ray colors (HR4). We define an heavily obscured region $\left(F(2-10 \mathrm{keV}) /\left(v_{25} F_{25}\right)<0.02\right.$ and HR4 $\left.>-0.2\right)$ where Compton-thick AGN are typically found. After cross-correlating the IRAS Point Source Catalog with the bright and hard $\left(F(4.5-12 \mathrm{keV})>10^{-13} \mathrm{erg} \mathrm{cm}^{-2} \mathrm{~s}^{-1}\right)$ end of the $2 X M M-N e w t o n$ catalog, we find 43 Compton-thick AGN candidates. Through a detailed X-ray spectral analysis (presented in a companion paper) we have found that about $84 \%$ of them are Comptonthick AGN. Twenty percent of the selected Compton-thick are newly-discovered ones. For comparison, the efficiency in finding Compton-thick AGN using other X-ray-to-mid-infrared diagnostic ratio (e.g. $L_{\mathrm{X}} / L_{6 \mu \mathrm{m}}$ ) is $50 \%$ and in an hard X-ray flux-limited survey is about $6 \%$. We have estimated also the completeness of the method that turns out to be of the order of $95 \%$.

After having taken into account selection effects, we have estimated the surface density of Compton-thick AGN down to the IRAS PSC catalogue flux limit $\left(F_{25}=0.5 \mathrm{Jy}\right)$ and we have compared it with that obtained from an hard X-ray survey performed with Swift-BAT (Burlon et al. 2011; Ajello et al. 2012). We find $\rho^{\mathrm{CT}} \sim 3 \times 10^{-3} \mathrm{src} \mathrm{deg}^{-2}$ that is a factor 4 above the density computed in the hard X-ray surveys. We find that this difference is, at least in part, ascribed to a significant contribution $(\sim 60-90 \%)$ of the star-forming activity to the total $25 \mu \mathrm{m}$ emission for the sources in our sample. By considering only the $25 \mu \mathrm{m}$ AGN emission, we estimate a surface density of Compton-thick AGN consistent with the results found with Swift-BAT.

Finally, we estimate the co-moving space density of Compton-thick AGN with $L_{\mathrm{X}}>10^{43} \mathrm{erg} \mathrm{s}^{-1}$ in a redshift range of 0.004-0.06 $\left(\Phi_{\mathrm{C}-\text { thick }} \sim\left(3.5_{-0.5}^{+4.5}\right) \times 10^{-6} \mathrm{Mpc}^{-3}\right)$. The prediction for Compton-thick AGN based on the synthesis model of the X-ray background in Gilli et al. (2007) is consistent with this value, while the prediction from Treister et al. (2009a) is lower.

Acknowledgements. The authors acknowledge financial support from ASI (grant No. I/088/06/0, COFIS contract and grant No. I/009/10/0). The authors thanks C. Vignali for insightful suggestions and V. La Parola and G. Cusumano for useful discussions about the BAT data. This research made use of the Simbad database and of the NASA/IPAC Extragalactic Database (NED). We would like to thank the anonymous referee for the useful and constructive comments which improved the quality of the paper.

\section{References}

Ajello, M., Alexander, D. M., Greiner, J., et al. 2012, ApJ, 749, 21 Alexander, D. M., Bauer, F. E., Brandt, W. N., et al. 2011, ApJ, 738, 44
Antonucci, R. 1993, ARA\&A, 31, 473

Avni, Y., \& Bahcall, J. N. 1980, ApJ, 235, 694

Awaki, H., Terashima, Y., Higaki, Y., \& Fukazawa, Y. 2009, PASJ, 61, 317

Ballo, L., Braito, V., Della Ceca, R., et al. 2004, ApJ, 600, 634

Bianchi, S., Piconcelli, E., Chiaberge, M., et al. 2009, ApJ, 695, 781

Braito, V., Della Ceca, R., Piconcelli, E., et al. 2004, A\&A, 420, 79

Braito, V., Reeves, J. N., Della Ceca, R., et al. 2009, A\&A, 504, 53

Brightman, M., \& Nandra, K. 2011, MNRAS, 414, 3084

Burlon, D., Ajello, M., Greiner, J., et al. 2011, ApJ, 728, 58

Caccianiga, A., Severgnini, P., Braito, V., et al. 2004, A\&A, 416, 901

Caccianiga, A., Severgnini, P., Della Ceca, R., et al. 2007, A\&A, 470, 557

Coziol, R., Torres, C. A. O., Quast, G. R., Contini, T., \& Davoust, E. 1998, ApJS, 119,239

Cusumano, G., La Parola, V., Segreto, A., et al. 2010, A\&A, 524, A64

Daddi, E., Alexander, D. M., Dickinson, M., et al. 2007, ApJ, 670, 173

Della Ceca, R., Ballo, L., Tavecchio, F., et al. 2002, ApJ, 581, L9

Della Ceca, R., Maccacaro, T., Caccianiga, A., et al. 2004, A\&A, 428, 383

Della Ceca, R., Severgnini, P., Caccianiga, A., et al. 2008a, Mem. Soc. Astron. Ital., 79, 65

Della Ceca, R., Caccianiga, A., Severgnini, P., et al. 2008b, A\&A, 487, 119

Draper, A. R., \& Ballantyne, D. R. 2010, ApJ, 715, L99

Georgantopoulos, I., Rovilos, E., Akylas, A., et al. 2011, A\&A, 534, A23

Gilli, R., Comastri, A., \& Hasinger, G. 2007, A\&A, 463, 79

Gilli, R., Vignali, C., Mignoli, M., et al. 2010, A\&A, 519, A92

Guainazzi, M., Matt, G., \& Perola, G. C. 2005, A\&A, 444, 119

Fiore, F., Grazian, A., Santini, P., et al. 2008, ApJ, 672, 94

Heckman, T. M., Armus, L., Weaver, K. A., \& Wang, J. 1999, ApJ, 517, 130

Helou, G., \& Walker, D. W. 1988, Infrared astronomical satellite (IRAS) catalogs and atlases, 7, 1

Horst, H., Gandhi, P., Smette A., \& Duschl W. J. 2008, A\&A, 479, 389

Hunt, L. K., \& Malkan, M. A. 1999, ApJ, 516, 660

Iwasawa, K., Sanders, D. B., Evans, A. S., et al. 2005, MNRAS, 357, 565

Magdziarz, P., \& Zdziarski, A. A. 1995, MNRAS, 273, 837

Maiolino, R., Salvati, M., Bassani, L., et al. 1998, A\&A, 338, 781

Maiolino, R., Comastri, A., Gilli, R., et al. 2003, MNRAS, 344, L59

Malizia, A., Stephen, J. B., Bassani, L., et al. 2009, MNRAS, 399, 944

Marconi, A., Risaliti, G., Gilli, R., et al. 2004, MNRAS, 351, 169

Matt, G., Pompilio, F., \& La Franca, F. 1999, New A, 4, 191

Miyawaki, R., Makishima, K., Yamada, S., et al. 2009, PASJ, 61, 263

Moshir, M. 1991, J. British Interplan. Soc., 44, 495

Murphy, K. D., \& Yaqoob, T. 2009, MNRAS, 397, 1549

Polletta, M. D. C., Wilkes, B. J., Siana, B., et al. 2006, ApJ, 642, 673

Polletta, M., Tajer, M., Maraschi, L., et al. 2007, ApJ, 663, 81

Ptak, A., Heckman, T., Levenson, N. A., Weaver, K., \& Strickland, D. 2003, ApJ, 592, 782

Ranalli, P., Comastri, A., \& Setti, G. 2003, A\&A, 399, 39

Rigby, J. R., Diamond-Stanic, A. M., \& Aniano, G. 2009, ApJ, 700, 1878

Risaliti, G., Elvis, M., Fabbiano, G., Baldi, A., \& Zezas, A. 2005, ApJ, 623, L93

Rush, B., Malkan, M. A., \& Spinoglio, L. 1993, ApJS, 89, 1

Sakai, S., \& Madore, B. F. 1999, ApJ, 526, 599

Sanders, D. B., Mazzarella, J. M., Kim, D.-C., Surace, J. A., \& Soifer, B. T. 2003, AJ, 126, 1607

Severgnini, P., Caccianiga, A., Braito, V., et al. 2003, A\&A, 406, 483

Severgnini, P., Caccianiga, A., \& Della Ceca, R. 2008, 8th National Conference on AGN, held in Torino, Italy, May 19-22, ed. L. Lanteri, C.M. Raiteri, A. Capetti, \& P. Rossi, Available online at agn8. oato.inaf.it

Severgnini, P., Caccianiga, A., Della Ceca, R., et al. 2011, A\&A, 525, A38

Shang, Z., Brotherton, M. S., Wills, B. J., et al. 2011, ApJS, 196, 2

Tajer, M., Polletta, M., Chiappetti, L., et al. 2007, A\&A, 467, 73

Teng, S. H., Veilleux, S., Anabuki, N., et al. 2009, ApJ, 691, 261

Tozzi, P., Gilli, R., Mainieri, V., et al. 2006, A\&A, 451, 457

Treister, E., Urry, C. M., \& Virani, S. 2009a, ApJ, 696, 110

Treister, E., Cardamone, C. N., Schawinski, K., et al. 2009b, ApJ, 706, 535

Trippe, M. L., Reynolds, C. S., Koss, M., Mushotzky, R. F., \& Winter, L. M. 2011, ApJ, 736, 81

Watson, M. G., Schröder, A. C., Fyfe, D., et al. 2009, A\&A, 493, 339 